\title{
Prodromes as Predictors of Hereditary Angioedema Attacks
}

\author{
Avner Reshef ${ }^{1}$, Iris Leibovich-Nassi ${ }^{1}$, Raz Somech $\mathrm{R}^{2}$, and Hava Golander ${ }^{3}$ \\ ${ }^{1}$ Barzilai Medical Centre Ashkelon \\ ${ }^{2}$ Sheba Medical Center \\ ${ }^{3}$ Tel Aviv University Sackler Faculty of Medicine
}

November 8, 2021

\section{Letter to the Editor}

\section{Prodromes as Predictors of Hereditary Angioedema Attacks}

\section{To the Editor}

Hereditary Angioedema (HAE) is a rare genetic disorder, expressed by recurring bouts of edema (swelling) in various organs and tissues. ${ }^{1}$ Pre-attack perceptions (Prodromes) are frequently reported by HAE patient's but since they are loosely defined they pose a clinical condundrum. ${ }^{2-3}$ Prodromes are subjective and objective signs and symptons, preceding attacks by several hours (Table S1). ${ }^{3}$ They may represent an early pathophysiologic events invlovling the bradykinin-forming cascade and the vascular endothelium. ${ }^{4-5}$ HAE patients may use these portending perceptions to predict oncoming attacks. ${ }^{2-3,6}$

Since specific research instruments are not available, prodromes have not been properly investigated by a methodical manner and their predictive power as an early warning sign is unknown. In view of these unmet needs, we constructed and validated a new HAE specific Patient-reported Outcome (PRO) instrument, capable of assessing the predictive power of prodromes. ${ }^{7}$

The present study is based on reports of 66 HAE patients (Table 1). Prodromes and attacks in relevant body locations (domains) were designated a 'cluster'. ${ }^{2,6}$ Accordingly, 'abdominal cluster' represents all recognizable gastrointestinal (GI) signs and symptoms. This method is easy to comprehend and amenable to statistical analysis. ${ }^{7}$ Clusters included: facial, oropharyngeal, abdominal, extremities (skin) and urogenital domains (Figure S1). They were evaluated by four scalable dimensions: pain, severity, impairment and functionality. Patients rated their recent experience with prodromes and attacks on a Visual Analog Scale (VAS). We received 223 patient reports on attacks, with $92 \%$ experiencing a prodrome before at least one attack, and $64 \%$ said they are able to predict an oncoming attack by a prodrome. We found significant and consistent differences between dimensions of prodromes and attacks across all clusters. Mean severity of prodromes was approximately half of attacks across all pre-specified clusters (Figure 1). Prodromes could be discriminated from attacks across all clusters, supporting our hypothesis that patients can distinguish between these two events (Tables S2, S3). Highly significant correlations were found between the dimensions and intensity of prodromes and attacks (Figure S2). We also proved that personal variables (i.e. experience and duration of illness) influences patients' perception of a prodrome. ${ }^{7}$ Sensitivity of prodromes as a predictor of attack was $89.5 \%$ and Specificity $63.1 \%$.

These results indicate that HAE patients can distinguish prodromes from attacks and that prodromes fortell subsequent attack location and intensity. Although prodromes and attacks are unpredictable and vary in their manifestations, experienced patients are more attentive to their body cues and capable of finding consistency and repetitiveness in prodromes. ${ }^{8}$ Patients can better cope with the challenges of the disease by using prodromes as an early warning sign and take preventative actions to mitigate attacks. Training 
patients to recognize early prodromes may be adopted as an efficient strategy for timing self-administration of rescue medication. ${ }^{9}$

We propose that a careful selection of relvant clusters and scaleable dimensions authentically reflect the patients' perception of disease severity and its impact on real-life illness prespective. The new instrument can be applied to other diseases with relapsing-remitting pattern, whose attacks are also antedated by prodromes, such as Epilepsy, Migraine and FMF, allowing fast deployment of therapeutic strategies to preempt attacks. ${ }^{10}$

In conclusion, we found that by using a disease specific tool, HAE patients can distinguish prodromes from attacks and predict oncoming attacks. Personal experience is a crucial factor in the patients' perception of a prodrome and ability to respond. This study adds to our knowledge on prodromes and their importance as attack-predicting harbingers. Early detection an attack is expected to assist in clinical decision-making regrading the timing of treatment and may shorten or even avert the attacks. Evaluating prodromes can be helpful in the management of HAE and other chronic illnesses with undulating course.

\section{REFERENCES}

1. Busse PJ, Christiansen SC. Hereditary Angioedema. N Engl J Med.2020;382:1136-48.

2. Reshef A, Prematta M, Craig TJ. Signs and symptoms preceding acute attacks of hereditary angioedema: results of three recent surveys. Allergy Ast Proc. 2013;34(3):261-266.

3. Leibovich-Nassi I, Reshef A. The Enigma of Prodromes in Hereditary Angioedema (HAE). Clin Rev Allergy Immunol. 2021;61(1):15-28.

4. Kaplan AP, Joseph K. Pathogenesis of Hereditary Angioedema: The Role of the Bradykinin-Forming Cascade.Immunol Allergy Clin N Am. 2017;37(3):513-25.

5. Luyasu S, Charignon D, Ponard D, Drouet C, Ghannam A. Angioedema: Systemic activation process during prodromes. Ann Allergy Ast Immunol. 2018;121(2):248-249.

6. Prematta MJ, Bewtra AK, Levy RJ, Wasserman RL, Jacobson KW, Machnig T, et al. Per-attack reporting of prodromal symptoms concurrent with C1-inhibitor treatment of hereditary angioedema attacks. Adv Ther. 2012;29(10):913-922.

7. Leibovich-Nassi I, Golander H, Somech R, Har-Even D, Reshef A. New Instrument for the Evaluation of Prodromes and Attacks of Hereditary Angioedema (HAE-EPA). Clin Rev Allergy Immunol.2021;61(1):29-39.

8. Barlow JH, Cullen A, Rowe IF. Comparison of knowledge and psychological well-being between patients with a short disease duration and patients with more established rheumatoid arthritis.Pat Edu Counsel. 1999; 38:195-203.

9. Maurer M, Aberer W, Bouillet L et al. Hereditary angioedema attacks resolve faster and are shorter after early icatibant treatment.PLoS One. 2013;8(2):e53773.

10. Babaoglu H, Varan O, Kucuk $\mathrm{H}$ et al. On demand use of anakinra for attacks of familial Mediterranean fever (FMF).Clin Rheumatol. 2019; 38(2):577-581.

Iris Leibovich-Nassi ${ }^{1,2}$ https://orcid.org/0000-0002-0097-4919

Avner Reshef ${ }^{1}$ https://orcid.org/0000-0002-3324-7072

Raz Somech ${ }^{3}$ https://orcid.org/0000-0001-6648-9425

Hava Golander ${ }^{2}$ https://orcid.org/0000-0002-8457-8736

Affiliations: ${ }^{1}$ Barzilai University Medical Center, Ashkelon, Israel, ${ }^{2}$ Sackler school of Medicine, Tel Aviv University, Israel, ${ }^{3}$ Safra Pediatric Medical Center, Ramat Gan, Israel

Corresponding author: Avner Reshef, email: Dr. Avner Reshef < aresh@netvision.net.il>

Keywords: attacks, domains, dimensions, hereditary angioedema, prodromes

Conflict of interst: None declared 
Declarations/Grants: ILN received the Stanley Steyer Graduate Scholarship Fund, Tel Aviv University and CSL Behring Fellowship Fund

No conflict of interest declared

Acknowledgments: We thank our colleagues from other HAE centers in Israel and the Israeli HAE patient organization (EDEMA) for their collaboration. We thank Mr. Dov Har-Even of Bar-Ilan University, for his excellent guidance and statistical evaluation.

Supporting information: Additional supporting information may be found online in the Supporting Information section.

Table 1: Disposition of patients in the study

\begin{tabular}{|c|c|c|}
\hline Variable & Category & Value (\%) \\
\hline Total number & & $N=66$ \\
\hline Age $(y s)$ & $\mathrm{M} \pm$ SD Mean Range & $32.4 \pm 16.430 .0 \quad 10-70$ \\
\hline Age Groups & $\begin{array}{l}10-20 \text { 21-30 } 31-40 \quad 41-50 \quad 51-60 \\
61-70\end{array}$ & $\begin{array}{l}19(28.8) 11(16.7) 14(21.2) 9 \\
(13.6) 7(10.6) 6(9.1)\end{array}$ \\
\hline Gender & Male Female & $27(40.9) 39(59.1)$ \\
\hline Type of HAE & Type I Type II & $60(90.9) 6(9.1)$ \\
\hline Family history & Yes No & $61(92.4) 5(7.6)$ \\
\hline Age at $1^{\text {st }}$ attack (ys) & $\mathrm{M} \pm$ SD Range & $9.8 \pm 7.21-48$ \\
\hline Age at diagnosis (ys) & $\mathrm{M} \pm \mathrm{SD}$ Range & $14.5 \pm 12.31-48$ \\
\hline Experience in HAE (ys) & $\mathrm{M} \pm \mathrm{SD}$ Range & $25.9 \pm 19.21-66$ \\
\hline Country of birth & Israel Europe America & $55(83.3)(12.2) 83(4.5)$ \\
\hline HAE in the family* & $\begin{array}{l}\text { Parents Siblings Grandparents } \\
\text { Uncles }\end{array}$ & $\begin{array}{l}49(74.2) 40(60.6) 32(48.5) 20 \\
(30.3)\end{array}$ \\
\hline Triggers** & $\begin{array}{l}\text { Injury Stress Pressure, } \\
\text { vibration Unknown Other } \\
\text { Menstruation Fever Diarrhea } \\
\text { Medicines }\end{array}$ & $\begin{array}{l}46(69.7) 31(46.9) 24(36.4) 23 \\
(34.8) 18(27.3) 16(24.2) 16 \\
(24.2) 14(21.2) 12(18.2)\end{array}$ \\
\hline $\begin{array}{l}\text { Prodrome } \\
\text { (Pre-attack) }\end{array}$ & $\begin{array}{l}\text { None At least once Sometimes } \\
\text { Can predict attack }\end{array}$ & $\begin{array}{l}11(16.6) 55(83.3) 53(80.3) 42 \\
(63.6)\end{array}$ \\
\hline
\end{tabular}

* Each patient may have more than one affected family member ** Each patient may have experienced more than one trigger of attack

Legend to Table 1. Disposition of patients in the study

Patient's demographics and their past medical history of 66 HAE patients included in the study, is shown on this table. Patients were requested to fill a structured questionnaires (HAE-EPA) relating to their demographic and past medical history and experience with prodromes and attacks (see Methods).

Figure 1. Dimensions of prodromes and attacks according to body locations

[CHART][CHART][CHART][CHART][CHART]

Legend: Attack Prodrome

Legend to Figure 1. Dimensions of prodromes and attacks according to body locations

HAE patients were asked to rate their past experience with prodromes and attacks during the previous 6 month, on a visual analog scale (VAS) ranging from 0 to $10 \mathrm{~cm}$. Mean VAS of 223 attacks, involving different body locations (clusters) are illustrated. All patients reported at least one abdominal attack during 
the respective period. Intensities of the prodromes were significantly less than those of the attacks, as demonstrated for each scalable dimension (pain, severity, impairment, functionality). Orange bars = attacks, blue bars $=$ prodromes 